\title{
Tri-level Thresholding using Invasive Weed Optimization based on Nonextensive Fuzzy Entropy
}

\author{
Cao Binfang ${ }^{1}$, Li Jianqi ${ }^{2}$ and Nie Fangyan ${ }^{3}$ \\ ${ }^{1}$ College of Physics and Electronics Science, \\ Hunan University of Arts and Science, Changde 415000, China \\ ${ }^{2}$ College of Electrical and Information Engineering, \\ Hunan University of Arts and Science, Changde 415000, China \\ ${ }^{3}$ College of Computer Science and Technology, \\ Hunan University of Arts and Science, Changde 415000, China \\ cao_bf@163.com,li_jianqi@126.com,niefyan@163.com
}

\begin{abstract}
This study presents a tri-level thresholding method for image segmentation with invasive weed optimization (IWO) algorithm. The objective of the proposed approach is to handle the nonextensivity and vagueness of image in segmentation, in the meanwhile to reduce the computation time. In this study, the histogram of image is converted to fuzzy domain by membership function firstly. Then the thresholding method is constructed through maximizing the sum of nonextensive entropy of subsets of the each part of fuzzy histogram. The IWO algorithm is used to search the optimal thresholds to reduce the computation time in the new method. Experiments on synthetic and real-world images are given to demonstrate the effectiveness of the proposed approach compared with the other methods.
\end{abstract}

Keywords: image thresholding; nonextensivity; vagueness; entropy; invasive weed optimization

\section{Introduction}

Image segmentation is considered as an important basic operation for meaningful analysis and interpretation of image acquired [1]. The success of an image analysis system depends on the quality of segmentation [2]. Among the existing segmentation techniques, thresholding is one of the most popular approaches in terms of simplicity, robustness, and accuracy [2]. It is based on the assumption that the objects can be distinguished by their gray levels. The optimal thresholds are those permitting the distinction of different objects from each other or different objects from the background. A lot of algorithms for image thresholding can be found in the literature [3-6].

In general, all thresholding methods work very well for a well-defined image which histogram has a deep valley between two peaks. However, a great deal of images is usually ill defined, i.e. they are corrupted by noise and/or irregularly illuminated, which leading to a multimodal histogram, there is no clear separation between object and background. In these cases, the ordinary thresholding approaches perform poorly or even fail. Fuzzy theory can be used to deal with these ambiguity and uncertainty in image thresholding, and many thresholding methods have proposed based on fuzzy sets theory in recent years [4-6]. In addition, the image is a typical physical system with nonextensive characteristics [7]. However, the ordinary thresholding methods often 
neglect the effects of nonextensive information on threshold selection, and thus no good results can be obtained sometimes [8]. Nonextensive entropy is an effective measure for physical system with nonextensive characteristics [9]. The nonextensive entropy is firstly used to image thresholding by Portes et al. [8], and has obtained better results [8, $10]$.

For handling the nonextensivity and vagueness of image in a thresholding method at the same time, a new tri-level thresholding method is presented based on nonextensive entropy and fuzzy sets theory in this paper. The new method involves the optimization of combination of fuzzy membership functions' parameters in the process of thresholds selection, and this process is very time-consuming. The invasive weed optimization (IWO) algorithm [11] is a function optimization algorithm, and its convergence performance is superior to the traditional optimization algorithm [11], so it has been applied in many engineering fields successfully [12-14]. To reduce the computation time, the IWO algorithm is used in the proposed new method. The experimental results show that the proposed approach is an effective method for image segmentation, and the computation time is satisfies the demand of engineering applications.

\section{Thresholding by Fuzzy Set and Nonextensive Entropy}

\subsection{Convert the Image Histogram to Fuzzy Domain}

Let $\boldsymbol{I}=\{f(x, y)\}$ denotes a digital image with size of $M \times N$ and $L$ grayscale levels, where $x=1, \ldots, M$ and $y=1, \ldots, N$. In addition, let $H=\{h(g) \mid g=0,1, \ldots, L-1\}$ denotes the normalized gray level histogram, and it can be calculated by $h(g)=n_{g} /(M \times N)$, where $n_{\mathrm{g}}$ denotes the number of pixels in the image with gray level $g$. Obviously, image is a crisp set data. The first step of image thresholding based on fuzzy sets theory is to convert the image histogram information to the fuzzy domain. Take into account the computational efficiency and the effectiveness of segmentation results, the following linear functions are used in the new method as the membership functions, for example

$$
\begin{aligned}
& \mu_{A}(g)=\left\{\begin{array}{lc}
1 & g \leq a \\
(g-c) /(a-c) & a<g \leq c \\
0 & g>c
\end{array}\right. \\
& \mu_{B}(g)= \begin{cases}0 & g \leq a \\
(g-a) /(c-a) & a<g \leq c \\
1 & g>c\end{cases}
\end{aligned}
$$

for bi-level thresholding, and

$$
\mu_{A}(g)=\left\{\begin{array}{lc}
1 & g \leq a \\
(g-b) /(a-b) & a<g \leq b \\
0 & g>b
\end{array}\right.
$$




$$
\begin{aligned}
& \mu_{B}(g)=\left\{\begin{array}{lc}
0 & g \leq a \\
(g-b) /(b-a) & a<g \leq b \\
1 & b<g \leq c \\
(g-d) /(c-d) & c<g \leq d \\
0 & g>d
\end{array}\right. \\
& \mu_{C}(g)= \begin{cases}0 & g \leq c \\
(g-c) /(d-c) & c<g \leq d \\
1 & g>d\end{cases}
\end{aligned}
$$

for tri-level thresholding. Where, $\boldsymbol{A}, \boldsymbol{B}$ and $\boldsymbol{C}$ denote the fuzzy subsets of image fuzzy domain; $a, b, c$ and $d$ denote the parameters of membership functions, and $0<a<c<255$ (for bi-level thresholding) and $0<a<b<c<d<255$ (for tri-level thresholding). The intersection(s) of the membership functions is (are) selected as the optimal threshold(s), namely, $t=(a+c) / 2$ (bi-level thresholding), or $t=((a+b) / 2,(c+d) / 2)$ (tri-level thresholding).

\subsection{Thresholding through Nonextensive Fuzzy Entropy}

According to the pseudo additivity rule of nonextensive entropy [15], the total nonextensive fuzzy entropy of image about fuzzy subsets can be defined as

$$
H(\mathbf{I})=H(\mathbf{A})+H(\mathbf{B})+(1-\alpha) \cdot H(\mathbf{A}) \cdot H(\mathbf{B})
$$

for bi-level thresholding, and

$$
\begin{aligned}
H(\mathbf{I})= & H(\mathbf{A})+H(\mathbf{B})+H(\mathbf{C})+(1-\alpha) \cdot[H(\mathbf{A}) \cdot H(\mathbf{B})+ \\
& H(\mathbf{A}) \cdot H(\mathbf{C})+H(\mathbf{B}) \cdot H(\mathbf{C})]+(1-\alpha)^{2} \cdot H(\mathbf{A}) \cdot H(\mathbf{B}) \cdot H(\mathbf{C})
\end{aligned}
$$

for tri-level thresholding. Where

$$
\begin{gathered}
H(\mathbf{X})=\frac{1}{1-q}\left(\sum_{g=0}^{L-1}\left(\mu_{\mathbf{X}}(g) \cdot h(g) / P_{\mathbf{X}}\right)^{q}-1\right) \\
P_{\mathbf{X}}=\sum_{g=0}^{L-1} \mu_{\mathbf{X}}(g) \cdot h(g)
\end{gathered}
$$

where the real number $q$ is an entropic index that characterizes the degree of nonextensivity; $H(\boldsymbol{X})$ denotes the tsallis entropy of image fuzzy subdomain $\boldsymbol{X}$, for bi-level thresholding, $\boldsymbol{X}=\boldsymbol{A}$ or $\boldsymbol{B}$, for tri-level thresholding, $\boldsymbol{X}=\boldsymbol{A}, \boldsymbol{B}$ or $\boldsymbol{C} ; H(\boldsymbol{I})$ denotes the total nonextensive fuzzy entropy of image $\boldsymbol{I}$. When $H(\boldsymbol{I})$ is maximized, the optimal threshold(s) can be obtained, namely

$$
t^{*}=\operatorname{argmax}(H(\mathbf{I}))
$$

In the limit $q \rightarrow 1$, the image nonextensive fuzzy entropy defined in this paper meets the fuzzy entropy defined by Zhao et al in [6]. 


\section{Thresholds Selection using Invasive Weed Optimization}

Since an exhaustive search for all fuzzy membership function parameter combinations is too costly, in this paper invasive weed optimization (IWO) algorithm is introduced into maximum nonextensive fuzzy entropy image segmentation to find the best fuzzy parameter combination adaptively.

The IWO algorithm developed by Mehrabian and Lucas [11] is a novel numerical stochastic optimization algorithm inspired from colonizing weeds. Weeds are plants growing in places we do not wish. They are very robust and adaptive to changes in the environment and are known as undesirable plants in agriculture. As a form of evolutionary algorithm, the IWO algorithm attempts to mimic the robustness, adaptation, and randomness of colonizing weeds. The basic characteristic of a weed is that it grows its population entirely or predominantly in a geographically specified area which can be substantially large or small. Initially a certain number of weeds are randomly spread over the entire search space. These weeds will eventually grow up and execute the following steps and the algorithm proceeds. The process flow of the IWO algorithm is outlined below [11-14]:

Step 1 Initialize a population: A population of initial solutions (seeds) is being dispread over the $n$ dimensional problem space with random positions. The population includes $m$ seeds, each seed denoted by $S_{i}=\left(x_{i 1}, x_{i 2}, \ldots, x_{i n}\right), i=1,2, \ldots, m$.

Step 2 The fitness of each individual seed is calculated according to the optimization problem, and the seeds grow to weeds able to produce new seeds.

Step 3 Reproduction: Each weed is ranked (increasing or decreasing) based on its fitness and produces new seeds depending upon its relative fitness in the population with respect to the best and worst fitness. The number of seeds produced by any weed varies linearly from min_seed to max_seed which is decided by the formula

$$
\text { number of seeds }=\frac{F_{i}-F_{\text {worst }}}{F_{\text {best }}-F_{\text {worst }}}\left(\text { max_seed }-m i n \_s e e d\right)+m i n \_s e e d
$$

In which min_seed for the worst member and max_seed for the best member in the population, $F_{i}$ is the fitness of $i$ th weed. $F_{\text {worst }}$ and $F_{\text {best }}$ denote the best and the worst fitness in the weed population. This step ensures that each weed take part in the reproduction process.

Step 4 Spatial dispersal: The generated seeds are normally distributed over the field with zero mean and a varying standard deviation of $\sigma_{i t e r}$ described by

$$
\sigma_{\text {iter }}=\frac{\left(\text { iter }_{\text {max }}-\text { iter }^{n}\right.}{\left(\text { iter }_{\text {max }}\right)^{n}}\left(\sigma_{\text {initial }}-\sigma_{\text {final }}\right)+\sigma_{\text {final }}
$$

where iter $_{\max }$ and iter are the maximum number of iteration and the current iteration number respectively. $\sigma_{\text {initial }}$ and $\sigma_{\text {final }}$ represent the pre-defined initial and final standard deviations. $n$ is the nonlinear modulation index and is generally assumed to be between 2 and 3.

Step 5 Competitive exclusion: The fitness of each seed produced in the above steps is calculated along with the parent weeds and by means of competitive exclusion. The seed-parent combinations that are least in fitness are eliminated and the number of weeds is limited to the maximum of number of weeds allowed. 
Step 6 The procedure is repeated at step 2 until the maximum number of iterations allowed by the user is reached or the optimized solution is obtained.

When we use IWO algorithm to find a combination of $(a, c)$ (for bi-level thresholding) or $(a, b, c, d)$ (for tri-level thresholding) such that $H(I)$ has the maximum value, we chose $x=(a, c)$ or $x=(a, b, c, d)$ as solution vector. It begins by generating $m$ seed randomly using $x_{i}^{j}=g_{\min }+r \cdot\left(g_{\max }-g_{\min }\right), i=1,2, \ldots, m, j=1,2$ or $j=1,2,3,4$ and $x_{i}^{1}=a, x$ ${ }_{i}^{2}=c$ or $x_{i}^{1}=a, x_{i}^{2}=b, x_{i}^{3}=c, x_{i}^{4}=d$ where $g_{\text {min }}$ and $g_{\text {max }}$ are the minimum and maximum grey level values of image. Notice that it is possible that the randomly generated individuals do not follow the increasing order $0<a<c<L$ (for bi-level thresholding) or $0<a<b<c<d<L$ (for tri-level thresholding). To resolve this problem, the elements of the individual vector are ordered by ascending firstly and then they will be put back the population to participate in iteration.

When the above scheme of IWO algorithm is applied to search the optimal parameters' combination of membership functions, a randomly population with size of $m$ is initialized firstly, then the IWO algorithm is iterated until the algorithm convergence. Finally, the optimal threshold(s) can be computed through the optimal parameters' combination.

\section{Experimental Results and Analysis}

We implement the proposed method in Matlab language with a Pentium(R) DualCore CPU E5500@2.80GHz and 2GB RAM. To evaluate the performance of the new method for image thresholding, we have implemented the proposed method on a variety of synthetic and real images. The results of the proposed method were compared with other widely used methods in the literature, i.e. the maximum entropy method proposed by Kapur et al. [3], the fuzzy entropy method proposed by Cheng et al. [5], the maximum fuzzy entropy method proposed by Zhao et al. [6], and the maximum nonextensive entropy method proposed by Portes et al. [8]. In addition, using many images, when $q$ is 0.7 our proposed method produced the best optimal thresholds, so the parameter $q$ is set to 0.7 in this paper. The parameters of IWO used for searching the optimal combination of parameters of membership function in proposed method are set as follows: the population size $m=30$, the maximum number of iterations iter $_{\text {max }}=100$, max_seed $=5$, min_seed $=1, \sigma_{\text {initial }}=0.1, \sigma_{\text {final }}=0.001$ and the nonlinear modulation index $n=3$.

\subsection{Performance Evaluation}

For evaluating the effectiveness of the different thresholding methods more objectively, we first conducted an experiment on a synthetic image for bi-level thresholding. In this study, we employ misclassification error (ME) measure [16] to evaluate the performances of the other methods. It regards image segmentation as a pixel classification process. ME reflects the percentage of background pixels wrongly assigned to foreground, and conversely, foreground pixels wrongly assigned to background. For the two-class segmentation problem, ME can be simply expressed as

$$
M E=1-\frac{\left|B_{o} \cap B_{T}\right|+\left|F_{O} \cap F_{T}\right|}{\left|B_{O}\right|+\left|F_{O}\right|}
$$

where $B_{O}$ and $F_{O}$ denote the background and foreground of the ground truth image, respectively; $B_{T}$ and $F_{T}$ indicate the background and foreground pixels in the segmented 
image, respectively, and $|\bullet|$ is the cardinality of a set $\bullet$. The value of ME varies from 0 for a perfectly classified image to 1 for a totally wrongly classified image. A lower value of ME means that the quality of the segmentation result is better.

The first image is shown in Figure 2(a), which is a simple synthetic image with $512 \times 512$ pixels and shows a Chinese character with gray level 80 on the background with gray level 190. The ideal segmentation of the synthetic image is shown in Figure 2(b). Gaussian noise with zeros mean and variance 0.005 is added to the original synthetic image as show in Figure 2(c). The histogram of noisy image is shown in Figure 2(d).

The segmented results obtained by the other methods corresponding to Figure 2(c) are shown in Figure 3. From the Figure 3, we can see that the results obtained by maximum fuzzy entropy (Figure 3(c)) and the proposed method (Figure 3(e)) are better than others results. The result obtained by Cheng et al's fuzzy method (Figure 3(b)) is very bad. There are more noise points in the results obtained by Kapur et al's method (Figure 3(a)), and the Portes et al.'s method (Figure 3(d)).

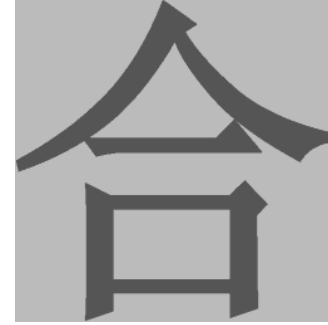

(a) Original image

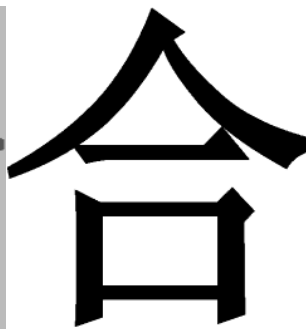

(b) ground truth image

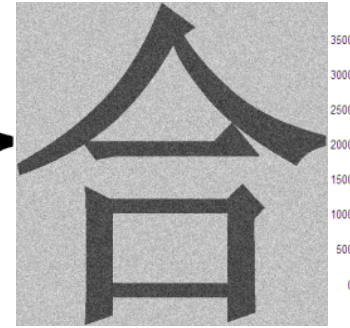

(c) noisy image

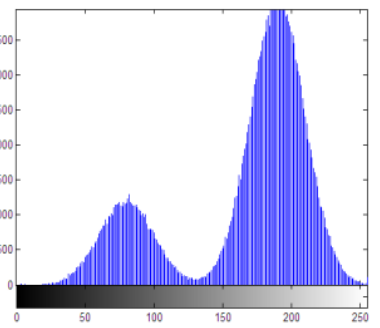

(d) histogram of noisy image

Figure 2. Synthetic Image

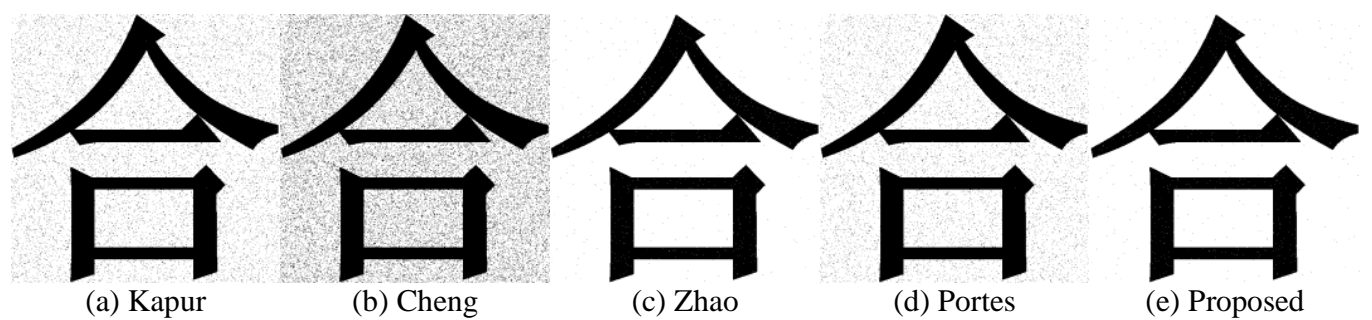

Figure 3. Thresholding Results of Synthetic Noisy Image by Different Methods

Table 1 shows the optimal thresholds, misclassified pixels, and ME values obtained by other methods. From the table 1, we can see that the number of misclassified pixels and the ME value obtained by proposed method is less than the results obtained by other methods.

Table 1. Performance Comparison of Segmentation on Synthetic Noisy Image

\begin{tabular}{cccccc}
\hline Method & Kapur & Cheng & Zhao & Portes & Proposed \\
\hline Threshold & 155 & 166 & 127 & 154 & 127 \\
Misclassified pixels & 8123 & 45447 & 663 & 7272 & 663 \\
ME & 0.0310 & 0.1734 & 0.0025 & 0.0277 & 0.0025 \\
\hline
\end{tabular}




\subsection{Experiments on Real Images}

To test the performance of proposed method on real images, we carried out a large number of experiments. For the limitation of space, the only two experimental results are listed. Figure 4 shows the real test images and their histograms. Figure 4(a) shows an 'MagrittePipe.tif' image with size $514 \times 670$ and its histogram is showed in Figure 4(b). Figure 4(c) is a 'robot.tif' image with size $227 \times 199$ and its histogram is showed in Figure 4(d). From the histograms of the test images, we can see that the target in 'MagrittePipe.tif' image can be separated by bi-level thresholding, while the object in the 'robot.tif' image can be separated by tri-level thresholding.

Figure 5 shows the segmented results of the 'eight.tif' image by all competing method. From Figure 5, we can see that the results by the Zhao's method (Figure 5(c)) and the proposed method (Figure 5(e)) are better than the results by other methods.

Figure 6 shows the results of the 'robot.tif' image. From the Figure 6, we can see that the results by the proposed method (Figure 6(e)) are better than the results by other methods.

Table 2 lists the thresholds and the running times by the different methods on real test images. Comparison of the histograms of real test images shown in the Figure 4 and the threshold(s) obtained by different thresholding methods in Table 2, we can see that the threshold(s) obtained by proposed method matched the valley of histogram better, while the threshold(s) obtained by other methods have more or less deviation. For the running time, the results of Kapur et al's method, and Portes et al's method are less than other methods on bi-level thresholding, while in the tri-level thresholding, the running time of the proposed method is less than that of other methods obviously, and it satisfies the requirement of engineering applications.

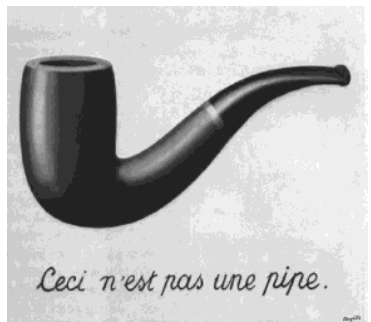

(a) MagrittePipe

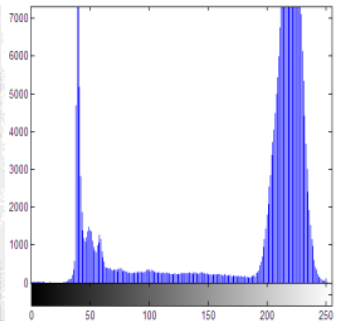

(b) histogram of (a)

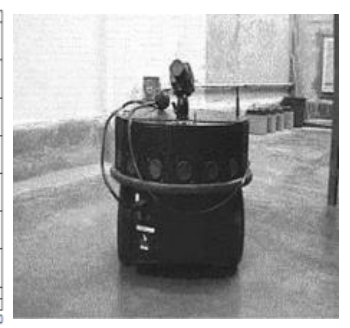

(c) robot

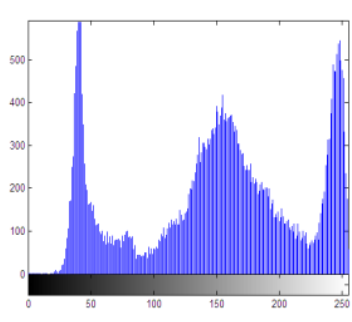

(d) histogram of (c)

Figure 4. Real Tested Images and their Histograms

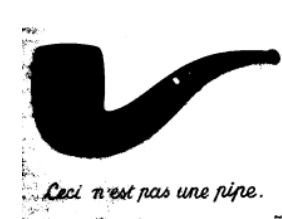

(a) Kapur

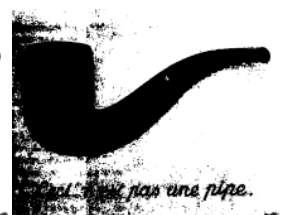

(b) Cheng

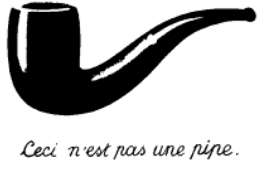

(c) Zhao

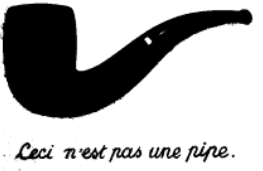

(d) Portes

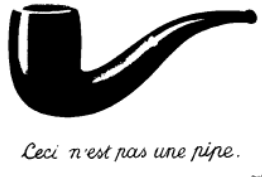

(e) Proposed

Figure 5. 2-level Segmented Results of Eight Image by Different Methods 


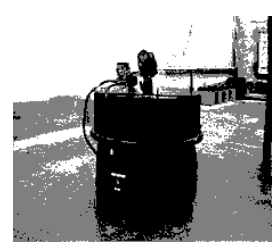

(a) Kapur

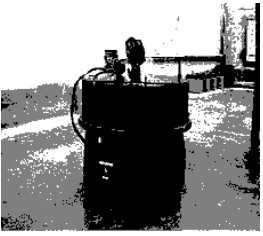

(b) Cheng

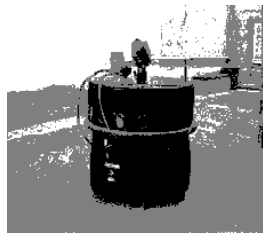

(c) Zhao

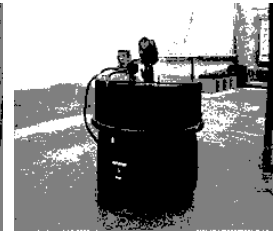

(d) Portes

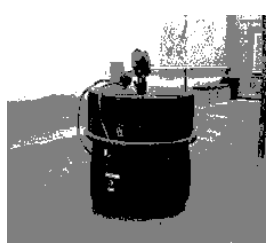

(e) Proposed

Figure 6. 3-level Thresholding Results of Robot Image by Different Methods

Table 2. Performance Comparison of Segmentation on Real Test Images

\begin{tabular}{ccccccc}
\hline \multicolumn{2}{c}{ Method } & Kapur & Cheng & Zhao & Portes & Proposed \\
\hline \multirow{2}{*}{ MagrittePipe.tif } & threshold & 197 & 209 & 133 & 193 & 134 \\
& time (s) & 0.1093 & 0.3962 & 0.3815 & 0.1095 & 0.3074 \\
\multirow{2}{*}{ robot.tif } & threshold & {$[123,182]$} & {$[129,179]$} & {$[68,190]$} & {$[117,182]$} & {$[78,200]$} \\
& time (s) & 10.1955 & 1.5301 & 1.9076 & 11.8471 & 1.0437 \\
\hline
\end{tabular}

From the above experimental results, we can see that the propose method is an effective method for image segmentation.

\section{Conclusion}

In order to handling the nonextensivity and vagueness information existed in image in thresholding, the study of this paper developed an effective thresholding method based on nonextensive entropy and fuzzy sets theory. When the optimal thresholds are searched in fuzzy domain, the time is costly. For reduce the search time, the invasive weed optimization algorithm is used in the presented method. The performance of the proposed method has been tested with various test images and compared with the other methods. The segmentation results obtained for various test images show that the proposed method could comprehensively outperform the competing method in terms of solution quality, computation efficiency and stability. Further research is to be carried out to test the feasibility of the proposed method for various types of image processing application.

\section{Acknowledgements}

The authors wish to thank the anonymous reviewers for the valuable comments and suggestions which helped to improve this paper. This research is supported by the Science and Technology Planning Project of Hunan Province, China (Grant No. 2012SK3131, 2014NK3125), and the Project of Hunan Provincial Natural Science Foundation, China (Grant No. 14JJ5008).

\section{References}

[1] M. Gong, Y. Liang, J. Shi, W. Ma, and J. Ma, "Fuzzy C-means clustering with local information and kernel Metric for image segmentation", IEEE Transactions on Image Processing, vol. 22, no. 2, (2013), pp. 573-584.

[2] M. Sezgin and B. Sankur, "Survey over image thresholding techniques and quantitative performance evaluation", Journal of Electronic Imaging, vol. 13, no. 1, (2004), pp. 146-165.

[3] J. N. Kapur, P. K. Sahoo and A.K.C. Wong, "A new method for gray-level picture thresholding using the entropy of the histogram", Computer Vision, Graphics, and Image Processing, vol. 29, no. 3, (1985), pp. 273-285.

[4] O. J. Tobias and R. Seara, "Image segmentation by histogram thresholding using fuzzy sets", IEEE Transactions on Image Processing, vol. 11, no. 12, (2002), pp. 1457-1465. 
[5] H. D. Cheng, J. R. Chen and J. Li, "Threshold selection based on fuzzy c-partition entropy approach", Pattern Recognition, vol. 31, no. 7, (1998), pp. 857-870.

[6] M. Zhao, A. M. N. Fu and H. Yan, "A technique of three-level thresholding based on probability partition and fuzzy 3partition”, IEEE Transactions on Fuzzy Systems, vol. 9, no. 3, (2001), pp. 469-479.

[7] I. Kilic and O. Kayacan, "A new nonlinear quantizer for image processing within nonextensive statistics", Physica A, vol. 381, (2007), pp. 420-430.

[8] M. Portes de Albuquerque, I. A. Esquef and A. R. Gesualdi Mello, "Image thresholding using Tsallis entropy", Pattern Recognition Letters, vol. 25, no. 9, (2004), pp. 1059-1065.

[9] C. Tsallis, "Possible generalization of Boltzmann-Gibbs statistics", Journal of Statistical Physics, vol. 52, no. 1/2, (1988), pp. 479-487.

[10] P. K. Sahoo and G. Arora, "Image thresholding using two-dimensional Tsallis-Havrda-Charvat entropy", Pattern Recognition Letters, vol. 27, no. 6, (2006), pp. 520-528.

[11] A. R. Mehrabian and C. Lucas, "A novel numerical optimization algorithm inspired from weed colonization", Ecological Informatics, vol. 1, no. 4, (2006), pp. 355-366.

[12] M. Ahmadi and H. Mojallali, "Chaotic invasive weed optimization algorithm with application to parameter estimation of chaotic systems", Chaos, Solitons \& Fractals, vol. 45, (2012), pp. 1108-1120.

[13] B. Saravanan, E.R. Vasudevan and D.P. Kothari, "Unit commitment problem solution using invasive weed optimization algorithm", International Journal of Electrical Power \& Energy Systems, vol. 55, (2014), pp. 21-28.

[14] P. Pahlavani, M. R. Delavar and A. U. Frank, "Using a modified invasive weed optimization algorithm for a personalized urban multi-criteria path optimization problem", International Journal of Applied Earth Observation and Geoinformation, vol. 18, (2012), pp. 313-328.

[15] A. M. Mathai and H. J. Haubold, "On generalized entropy measures and pathways", Physica A: Statistical Mechanics and its Applications, vol. 385, no. 2, (2007), pp. 493-500.

[16] S. T. Wang, F. L. Chung and F. S. Xiong, "A novel image thresholding method based on Parzen window estimate", Pattern Recognition, vol. 41, no. 1, (2008), pp. 117-129.

\section{Authors}

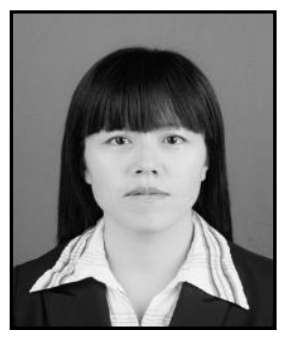

Cao Binfang, she was born in Shanxi province, China in 1979. She received her B.S. degree from Central South University, China in 2002 and her M. S. degree from Hunan University, China in 2007, respectively. Now, she is a Ph. D. candidate of Central South University. Her current research interests include image processing, modeling and control of complex industrial process.

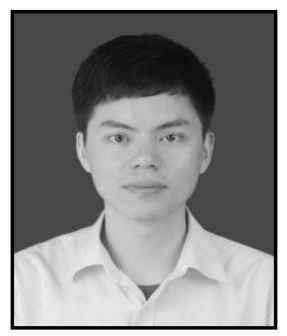

Li Jianqi, he was born in Hunan province, China in 1980. He received his Ph.D. degree in Control Science and Engineering from Central South University, China in 2013. His current research interests include intelligent information processing, image processing and pattern recognition.

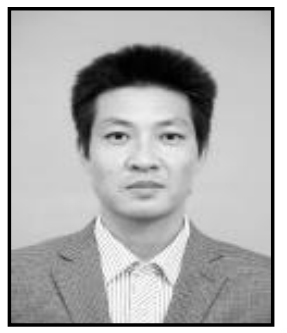

Nie Fangyan, he was born in Hunan province, China in 1977. He received his Ph.D. degree in Instrument Science and technology from Chongqing University, China in 2010. His current research interests include information acquisition and processing, image processing and pattern recognition. 
International Journal of Signal Processing, Image Processing and Pattern Recognition Vol. 7, No. 6 (2014) 